\title{
Educar al cuerpo dentro y fuera del aula. Análisis de dos experiencias en la Nueva Argentina de Perón
}

\section{Educate the body inside and outside the classroom. Analysis of two experiences in Perón's New Argentina}

\author{
Iván Pablo Orbuch \\ Orcid: http://orcid.org/0000-0001-7596-4611 \\ Instituto de Educación, Universidad Nacional de Hurlingham, Buenos Aires, \\ Argentina, ivan.orbuch@unahuredu.ar
}

Received on 07/05/2020 - Approved on 25/05/2020

\section{Resumen}

Luego de disensiones internas respecto a los modos de intervención estatal, se creó por decreto presidencial el Consejo Nacional de Educación Física en el año 1947. Tuvo entre sus propósitos principales la tarea de difundir la educación corporal en la población. Dos importantes iniciativas en ese sentido fueron los cuadernillos llamados Gimnasia compensatoria en el aula y Gimnasia para oficinas. En el primer caso, se trató de realizar actividades físicas a toda hora en la escuela, siendo novedoso el intento de hacer ejercicios en medio de las clases de todas las asignaturas escolares. Por su parte, la segunda propuesta nombrada buscó extender la influencia del organismo por fuera de los muros escolares. Un abordaje de ambas me permitirá a arribar a algunas conclusiones acerca de las características que adoptó la Educación Física en la Nueva Argentina de Perón.

Palabras claves: Consejo. Nacional. Educación. Física. Peronismo.

\begin{abstract}
After internal dissensions regarding the modes of state intervention, the National Council of Physical Education was created by presidential decree in 1947. Its main purposes were to spread body education among the population. Two important initiatives in this regard were the booklets called Compensatory gymnastics in the classroom and Gymnastics for offices. In the first case, an attempt was made to carry out physical activities at all times at school, with an innovative attempt to do exercises in the middle of classes for all school subjects. For its part, the second proposal named sought to extend the influence of the organism outside the school walls. An approach of both will allow me to reach some conclusions about the characteristics that Physical Education adopted in Perón's New Argentina.
\end{abstract}

Keywords: National. Council. Education. Physics. Peronism. 


\section{Introducción}

La fundación de entes gubernamentales destinados a regular y dirigir la educación corporal ocurrió tanto en los años previos al ascenso del peronismo a instancias de poder, como en los prolegómenos de su gobierno. Por caso, en la década de 1930 puede citarse a la Dirección General de Educación Física y Cultura creada por Manuel Fresco el 21 de julio de 1936 en la provincia de Buenos Aires. El 4 de junio de 1937 se erigió el Consejo Nacional de Educación Física. De acuerdo a lo planteado por Eduardo Galak (2012), el 17 de junio de 1938, y como contrapartida pedagógica, se creó la Dirección General de Educación Física bajo dependencia del Ministerio de Justicia e Instrucción Pública.

Este breve recorrido demuestra que antes de la llegada del peronismo al poder la cultura física tomó tanta importancia que se crearon varios organismos con la finalidad de regularla y difundirla.

Por su parte, en la primera etapa del gobierno de Juan Domingo Perón se hicieron palpables las discrepancias al interior de la coalición oficialista en lo concerniente al modo en que debía implementarse la Educación Física. Fue así que se presentaron tres propuestas legislativas. El 26 de septiembre de 1946, el Senador Nacional Diego Luis Molinari, presentó un proyecto de Ley, proponiendo la creación de la Dirección Nacional de Educación Física bajo la órbita del Ministerio de Guerra. La segunda de las iniciativas se dio el 23 de octubre de 1946 cuando el jujeño Manuel Sarmiento, proveniente de la Unión Cívica Radical Yrigoyenista, el porteño Antonio Benítez y el bonaerense José Emilio Visca, ambos integrantes del Partido Laborista elaboraron un proyecto de Ley con la finalidad de crear el Consejo Nacional de Educación Física y Medicina del Deporte en dependencia de la Secretaría de Salud Pública. Por último, el 5 de febrero de 1947, se presentó un proyecto para erigir el Consejo Nacional de Educación Física bajo la dependencia del Ministerio de Guerra.

En lo concerniente a la Dirección Nacional de Educación Física, se sostiene en sus fundamentos la imperiosa necesidad de su creación ya que Argentina se encontraba retrasada respecto a países como Estados Unidos, Brasil, México, Chile y Venezuela quienes contaban con organismos estatales que organizaban y coordinaban la Educación Física. Dado que esta era una parte central de la educación integral de los ciudadanos, el deber del Estado pasaba por fomentarla y regularla (Cámara de Diputados, 1946a).

El Consejo Nacional de Educación Física y Medicina del Deporte tuvo entre sus objetivos unir el aspecto sanitario con la educación corporal situándose dentro de la medicina social. Esta especialidad luchaba contra los factores indirectos de la enfermedad y mortalidad, dado que buscaba asegurar la prolongación de la vida útil del hombre. Vale la pena destacar que la intervención estatal en los procesos biológicos de las personas fue una constante en el plano internacional a mediados de siglo XX y fue definida por Michael Foucault como biopolítica. Abonando lo mencionado, en un trabajo realizado con Eduardo Galak (2017) ${ }^{1}$, sostenemos que:

\footnotetext{
Resulta significativo aclarar que este sentido respecto a lo político del peronismo puede ser leído en clave biopolítica pensada como régimen de la vida moderna. El modo de gobierno peronista expresado en los Planes Quinquenales de 1946-1951 y 1952-1955 refleja una explícita pretensión por
} 
gestionar centralizadamente la vida de la población, particularmente a través de institucionalizar -y, por ende, legitimar- prácticas sociales. (2017: 7)

La iniciativa legislativa planteaba que el presidente del Consejo Nacional de Educación Física y Medicina del Deporte tenía que ser un civil. Además, se abogaba por la obligatoriedad de la Educación Física en todos los niveles educativos, tanto "nacionales, municipales, o particulares" (Cámara de Diputados, 1946b). La formación profesional de los futuros docentes también quedaba en manos del Estado.

Por su parte, el Consejo Nacional de Educación Física debía quedar bajo dependencia del Ministerio de Guerra, colaborar con los equipos técnicos de las Fuerzas Armadas a fin de fijar normas para difundir la cultura física, ocuparse de lo relativo a la práctica e instrucción del tiro deportivo y proponer los planes de enseñanza para la pre y post conscripción (Cámara de Diputados, 1947a). Asimismo, debía dar las directivas "a las que ha de someterse la Educación Física por el personal de las organizaciones industriales y comerciales, de fábrica y de talleres, así como el de las reparticiones estatales" (1947a). Esto demuestra: por un lado, las intenciones de expandir la cultura física por fuera del sistema educativo y por el otro el propósito de dotarla de una impronta militarista.

En el Consejo Nacional de Educación Física se buscaba establecer la obligatoriedad de la práctica de la Educación Física en las escuelas primarias, secundarias, especiales y superiores sean nacionales, municipales o privadas (1947a). La citada exigencia contribuyó a dejar en claro la escasez de condiciones adecuadas en la mayoría de los establecimientos del país. Su falta de obligatoriedad hasta entonces, revelaba que la asignatura ocupaba aún un lugar subalterno en la escuela.

Las dilaciones en la sanción de estos proyectos de ley mencionados, influyeron para que Perón decida intervenir en el asunto. De hecho, el 10 de febrero de 1947 se dirigió a la Honorable Cámara de Diputados de la Nación solicitándoles que "se incluya entre los asuntos a considerar en las actuales sesiones extraordinarias, el Proyecto de Ley por el que se crea el Consejo Nacional de Educación Física" (Cámara de Diputados, 1947b) enviado apenas días antes, el 5 de febrero de ese año. Finalmente, el entonces presidente dispuso su creación por medio del decreto 34817 del 6 de noviembre de 1947. La existencia de distintos proyectos de leyes en un corto periodo reveló la presencia de tensiones al interior de la coalición gubernamental.

El Consejo Nacional de Educación Física puede interpretarse como un elemento de continuidad respecto a organismos gubernamentales erigidos con antelación, cuyo propósito era planificar la cultura física poblacional. No obstante, a los fines de esta investigación, el signo distintivo del nuevo ente radica en las rupturas propiciadas por su accionar visibles en la escala y masividad de sus políticas, puesto que estas se encontraron dirigidas a toda la población. Esto significará la intención de abarcar a todo el territorio nacional con contenidos, propuestas metodológicas, recursos humanos y materiales provistos por el Estado en sintonía con la mayor centralización propuesta por el gobierno peronista en otras esferas gubernamentales. 


\section{Gimnasia compensatoria en el aula}

En el año 1949 un cuadernillo elaborado por el Consejo Nacional de Educación Física llegó al ámbito escolar. Su título era Gimnasia Compensatoria en el aula. Su autor fue Alejandro Amavet quien cumplió un destacado papel en el campo de la Educación Física ${ }^{2}$. El folleto estuvo destinado a alumnos de segundo a sexto grado de la escuela primaria y se distribuyó en las escuelas de todo el país mediante la impresión de 10 mil ejemplares (Consejo Nacional de Educación Física, 1949). En su portada se aprecia a un alumno haciendo ejercicios en su pupitre.

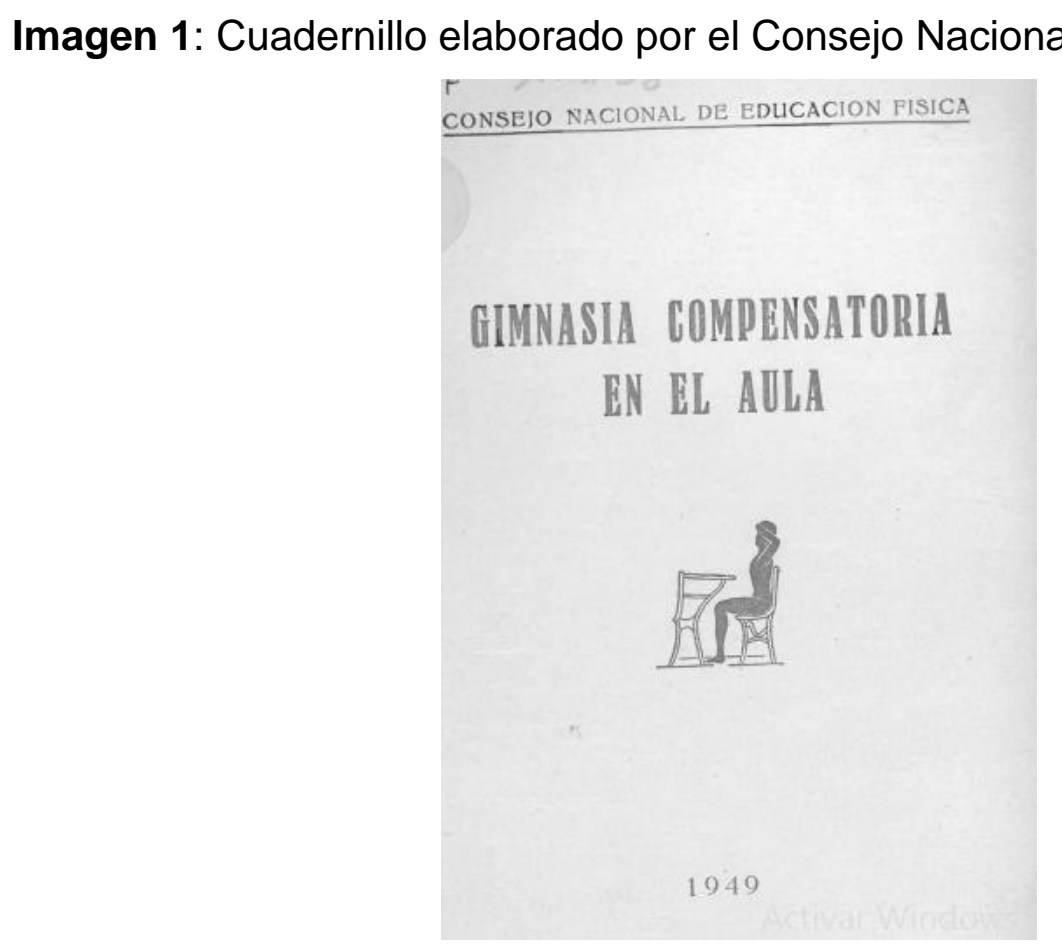

Fuente: Gimnasia Compensatoria en el aula. 1949

Precisamente esa era la idea del material: introducir la Educación Física en todas las asignaturas escolares, ya que su práctica diaria:

No interfiere en lo más mínimo en la enseñanza de las otras asignaturas, pues tres minutos restados a la hora escolar nada significan y reemplazan en forma útil un tiempo mucho mayor que se pierde en reconvenciones disciplinarias, que son las que realmente perjudican el normal desarrollo de aquellas. (1949: 3)

El cuadernillo intentó ser un firme aliado de la educación corporal que debían poseer los alumnos argentinos, demostrando el lugar protagónico que de a poco empezaba a tener en el sistema educativo. La Educación Física, desde esta lógica, puede pensarse como el dispositivo por excelencia de la educación del cuerpo. 
Imagen 2: Alunnos ejercitándose en clase

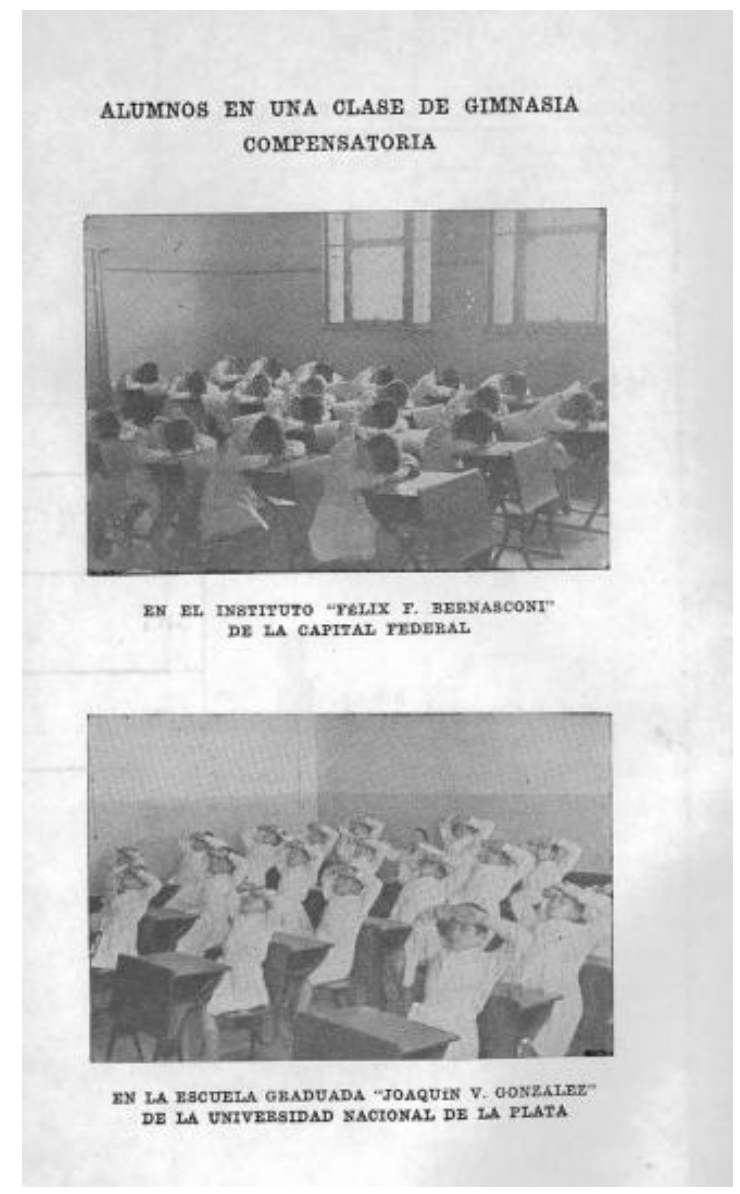

Fuente: Gimnasia Compensatoria en el aula. 1949

Las imágenes muestran un grupo de alumnos de nivel primario ejercitándose en clase en dos prestigiosas escuelas, El Instituto Félix Bernasconi del barrio de Parque Patricios sito en Capital Federal, y la Escuela Graduada Joaquín V. González, dependiente de la Universidad Nacional de La Plata. La trascendencia de esas instituciones educativas sirve como legitimadoras de las prácticas que se quieren implementar. Puede esgrimirse, en la percepción de cualquier directivo que tome contacto con este folleto, que si esas notables escuelas practicaban la gimnasia compensatoria no debían caber dudas de sus altos efectos benéficos.

El presente folleto tiene por objeto poner al alcance del docente un procedimiento simple pero eficaz para prevenir-y en todo caso contrarrestarlos vicios más comunes de la postura del educando cuyas causas deben atribuirse tanto a los bancos inapropiados, como a la inmovilidad forzada en el aula y a las técnicas de enseñanza de ciertas ramas intelectuales y manuales. (1949: 3)

Un objetivo explícito es el de normalizar hábitos y conductas a fin de lograr una homogeneización de la población escolar. Por su parte, otro concepto que recorre las páginas del citado documento es que debe desterrarse "la antinomia de lo físico con lo mental" (1949: 3). De ese modo las argumentaciones hacían hincapié en dejar en claro las potencialidades de este tipo de gimnasia que se 
haría a toda hora y en todas las clases a fin de dotar a los alumnos de una mejor postura ya que "sólo el cuerpo sigue siendo la envoltura ordinaria que protege a medias el precioso don del conocimiento y la sabiduría" (1949: 3)

Las ideas de una educación integral que contemple lo físico, lo intelectual y lo moral también pueden vislumbrarse en el presente documento del Consejo Nacional de Educación Física.

\begin{abstract}
Cierto es que para los defectos posturales se habla de gimnasia correctiva, como si esta especial terapéutica fuera tan fácil y conveniente de aplicar actualmente en la escuela, pero es necesario convenir que tales defectos tienen su origen por lo general en los malos hábitos de la actitud y que estos pueden fácilmente combatirse por quienes tienen la misión de educar, no unilateralmente, sino en toda la acepción de la palabra. Y si el maestro estudia para a su vez enseñar las asignaturas intelectuales más diversas, porque así lo exige su delicada y noble misión, también debe poseer el conocimiento y la práctica de todo aquello que beneficie al educando en lo que se refiere a su naturaleza física. Recién entonces podrá ostentar el honroso título de educador. (1949: 5)
\end{abstract}

El intento de posicionar a la Educación Física como una asignatura prestigiosa dentro del sistema escolar, del mismo modo que el llamamiento a la participación docente se observa con claridad. La conciencia de la responsabilidad inherente a la labor docente, "sabiamente administrada, le hará ver que no es el desarrollo más o menos perfecto del plan de estudio el desiderátum de la escuela, sino el alumno en su educación integral que es, en suma, el principio y el fin de la misma" (1949: 5). En esa educación integral que pregonaba el gobierno peronista el lugar de la Educación Física era central. Por eso no debe sorprender que a lo largo del documento se busque dejar en claro los efectos positivos tanto para la escuela, como para los docentes y los alumnos.

Sobre los estudiantes, la gimnasia compensatoria podía contribuir a crear los hábitos automáticos de una buena postura "liberándolo de reconvenciones verbales inoportunas" (1949). A su vez:

ayuda al crecimiento normal del niño. Beneficia el aparato cardiorespiratorio, sin alterar mayormente su ritmo normal. Actúa higiénicamente sobre su incontenible necesidad de movimiento al encauzar la actividad infantil al procurar la satisfacción subsiguiente. Actúa positivamente sobre la conducta normalizando la atención pata el aprendizaje de las asignaturas intelectuales. (1949: 5)

Desde esta perspectiva, la Educación Física "ya no será simplemente un saber de la normación sino de la normalización” (Crisorio, 2007: 73)

Respecto a la labor docente, la gimnasia compensatoria contribuía con numerosos aportes, subestimados por "la educación intelectualista en boga" (Consejo Nacional de Educación Física, 1949: 9). De ese modo:

Aseguraba una mejor fiscalización de la enseñanza que imparte a la dada, por la eliminación de la fatiga psicofísica que disminuye la atención y predispone a 
la indisciplina. Evita toda clase de reconvenciones verbales a los mal sentados o que adoptan posturas inconvenientes, Permite un mayor dominio disciplinario sobre la clase. Completa digna y eficazmente su acción de educador. (1949: 6)

En lo atinente a la institución escolar, la gimnasia compensatoria contribuye a normalizar, afianzar y consolidar la disciplina de todos los grados. Siguiendo los aportes de Somoza Rodríguez (2006), la mirada disciplinaria de la pedagogía implementada por el gobierno peronista se aprecia en los propósitos de este cuadernillo. De este modo la gimnasia compensatoria:

\begin{abstract}
Refuerza eficazmente la relativa eficiencia de la actual clase de Educación Física. Ya que esta necesita determinadas comodidades y elementos, de los que por ahora carece, para cumplir sus fines: la gimnasia compensatoria se hace diariamente sin necesidad de gimnasio, vestuario, vestimenta y ducha. Contribuye a dignificar la misión de la escuela transformándola realmente en el centro de formación integral de la niñez. (Consejo Nacional de Educación Física, 1949: 5)
\end{abstract}

Por cierto, para que todas estas ventajas pudieran materializarse era menester, desde la perspectiva de los funcionarios que pergeñaron este material, que los docentes se dieran cuenta de sus enormes potencialidades. Sólo así se podían adquirir resultados positivos. La constante recurrencia en la búsqueda de la ayuda del plantel docente, refleja que necesariamente hubo tensiones para que el peronismo imponga uniformemente sus criterios, en línea con lo planteado por Adriana Puiggrós y Jorge Bernetti (1993). El folleto dejaba en claro, ante la posible desconfianza del cuerpo magisterial, que el propósito no era formar gimnastas, sino que se buscaba formar el carácter de los argentinos. A tales efectos, era:

\begin{abstract}
Necesario dejar de considerar a la Educación Física como una asignatura de enseñanza ya que el alumno no viene a aprenderla sino a hacerla. Cuando falla este postulado esencial, el maestro trabaja sin interés y el aburrimiento alcanza a la mayoría de los alumnos. Por extensión la escuela resulta engañada y se utiliza a los niños para realizar inútiles remedos de desfiles y números anodino donde no hay arte y menos aún Educación Física. La gimnasia compensatoria tendrá la virtud de hacer conocer lo que es un educando en vías de formación y demostrar al maestro que así como puede obrarse sobre la inteligencia y sentimiento de los alumnos, puede actuarse con toda eficiencia sobre el cuerpo preservándolo, mejorándolo y embelleciéndolo. (Consejo Nacional de Educación Física, 1949: 7)
\end{abstract}

El documento realiza una directa crítica a los desfiles infantiles, asociado al tipo de Educación Física propiciada por los militares, si bien su autor era un docente con larga trayectoria como docente en al ámbito militar, lo que demuestra la complejidad del periodo. La distinción entre escuela teórica y escuela práctica, cara al ideario peronista, donde se busca formar un ciudadano activo y en movimiento se torna visible, del mismo modo que las reminiscencias de un discurso impregnado de tintes eugenésicos (Armus, 2016: 157). 
Se puede verificar que estos postulados están inmersos en la necesidad de generar una escuela activa que deje atrás las tradicionales características discursivas donde el docente se pasaba largos minutos explicando los temas.

La gimnasia compensatoria debía impartirse en conjunto bajo la dirección del propio maestro de grado, el que ulteriormente se haría reemplazar, para que sirva de estímulo, por aquellos alumnos que demuestren mayores disposiciones y aptitudes. Las indicaciones al modo de impartir la gimnasia compensatoria revelan que la misma debía practicarse:

a) Diariamente SIN EXCEPCIÓN a razón de una vez por cada hora escolar.

b) Con una duración no mayor de TRES MINUTOS por clase.

c) AL PROMEDIAR LA HORA DE CLASE, que es cuando comienza a manifestarse la necesidad de su aplicación. Si razones pedagógicas de otra índole aconsejan su postergación (no su adelanto o eliminación) será conveniente que su práctica se realice no lejos de la señal que marca la mitad de la hora escolar. (Consejo Nacional de Educación Física, 1949a: 9)

El folleto dedica numeroso espacio a enseñar la didáctica de los ejercicios a los docentes que se encargarían de llevar a la práctica este tipo de gimnasia, que es un claro intento de expandir la influencia de la Educación Física al resto de las asignaturas escolares. Empero, es marcado el tono exploratorio de las sugerencias a adoptar, seguramente como un modo de cooptar docentes para llevarlas adelante.

La correcta postura al sentarse es uno de los temas claves para la gimnasia compensatoria y en el cuadernillo se dedican numerosos gráficos y explicaciones para que los docentes puedan comprender la específica didactica que requería.

A sabiendas de las distintas lecturas que el plantel profesoral podía hacer del material se realizó una aclaración que buscaba a la vez tranquilizar y sumar adeptos para la implementación de la gimnasia compensatoria:

Para la confección de este plan elemental se ha tenido en cuenta, primordialmente, su valor de aplicación. Precisamente su sencillez está de acuerdo con lo factible y tanto el maestro que lo interprete y enseñe como el alumno que lo practique, no han de encontrar en él los inconvenientes de otras iniciativas de gran valor pedagógico para la escuela, pero cuya aplicación se ha visto trabada desde sus comienzos por pretender lo amplio y definitivo antes que lo simple y gradual, que el principio esencial de toda enseñanza. Cuando se aprecien los beneficios, los maestros serán los más grandes entusiastas propulsores de esta actividad. (1949: 19)

El derrotero de esta iniciativa, que distó de poder implementarse de modo acabado, da cuenta de las tensiones existentes entre las directivas emanadas y su efectiva puesta en funcionamiento (Ball, 1990). En efecto, puede apreciarse que la relación entre la macro política educativa y la realidad institucional escolar no se presentó exenta de conflictos. 


\title{
Gimnasia de Oficinas
}

Imagen 3: Portada del cuadernillo Gimnasia de Oficinas. 1950.

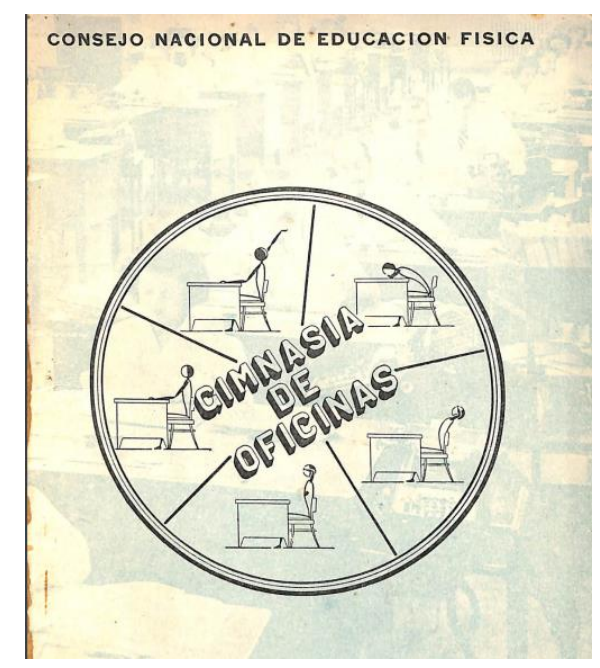

Fuente: Cuadernillo Gimnasia de Oficinas. 1950.

El folleto fue parte de las políticas públicas implementadas por el gobierno peronista en lo referido a diseminar la educación corporal en la población. Los datos disponibles respecto a su forma de distribución son escasos y sólo se menciona en el folleto que se imprimieron cinco mil ediciones, lo cual es un número considerable. Se trató de un segmento fuera del alcance de las políticas educativas, y por tanto puede afirmarse que fue novedosa la propuesta en tanto el gobierno peronista buscó adentrarse en el ámbito privado desde las políticas públicas, lo cual redundó en un interés por mejorar las condiciones de vida de la población, a tono con postulados biopolíticos (Foucault, 1992). En ese sentido, cabe destacar que en la primera página se hace referencia al derecho a la preservación de la salud, tal como se lee en la nueva Constitución sancionada en el año 1949. Allí se hace referencia a que el derecho a la salud física, está asociada a la salud moral, debiendo la sociedad y el Estado velar por ambas, lo que explicaría la injerencia estatal en el ámbito privado.

El capítulo 1, titulado generalidades, advierte sobre algunas extendidas percepciones que el cuadernillo se proponía desterrar. El énfasis en estas cuestiones es visible, como si el éxito de la medida dependiera de poder convencer a los oficinistas de las bondades de la cultura física.

\begin{abstract}
Si entráramos en una oficina común en plena actividad, haciendo abstracción completa de todo elemento de trabajo o producción, y nos concretáramos a observar exclusivamente y con criterio anatómico al numeroso personal absorbido por la tarea cotidiana, quedaríamos asombrados por la cantidad de interesantes comprobaciones y conclusiones que nos depararía tan particular examen. (Consejo Nacional de Educación Física, 1950: 3).
\end{abstract}

Muchas de esas conclusiones hacían referencia a la rutina laboral que era interpretada como perniciosa para la salud corporal y mental transformando a los hombres y mujeres en personas agobiadasLámina 4: que hacen su trabajo "como 
un mal necesario para subsistir" (1950: 3). Estas condiciones dieron lugar a la llamada "espalda de oficinas", que era la denominación con la que se conocía a la espalda curva, producto de las horas de trabajo en una misma posición. situación:

El cuadernillo prosigue explicando que se ha intentado poner fin a esta

Desde el taylorismo hasta la recreación y el empleo de las horas libres, desde la medicina del trabajo en sus fases preventivas de la higiene y la psicotécnica, y terapéuticas de las clínicas y hospitales, hasta las modernas legislaciones sociales, todas las técnicas adoptadas y ensayadas se han movido, por lo general, dentro de un respeto absoluto por el tiempo de trabajo, actuando ya sea antes o después de las horas dedicadas al mismo. (1950: 3).

La explicación hizo hincapié en que el citado excesivo respeto por el trabajo se tornó perjudicial puesto que contribuyó con un exponencial aumento de la fatiga muscular de todos los trabajadores de oficina.

Imagen 4: Oficina de una gran casa comercial de Estocolmo donde se realizaron por primera vez intervalos de gimnasia para oficinistas. 1950.

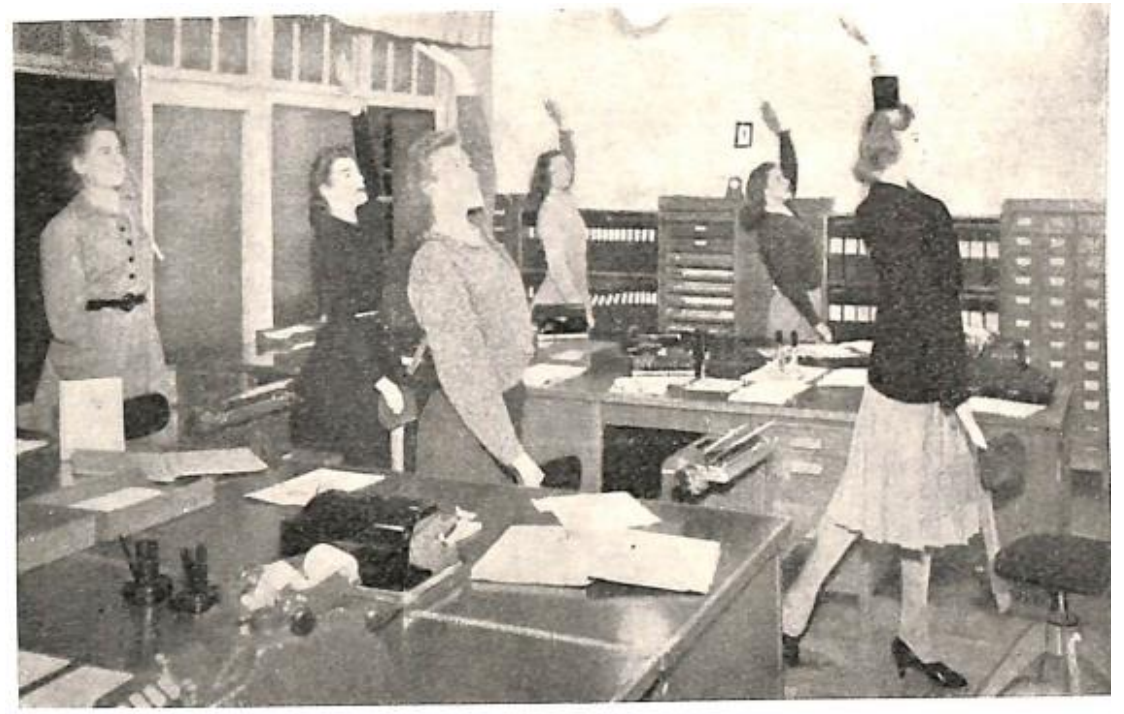

Ofieina de una gran casa eomercial de Estocolmo donde se realizaron por primera vez intervalos de gimasia

para ofieinistas

Fuente: Cuadernillo Gimnasia de Oficinas. 1950.

La gimnasia de oficinas según el folleto, "era un tipo profesional de gimnasia compensatoria que, desarrollada sin mayores exigencias disciplinarias y formales, obra a manera de derivativo psicofísico, descanso, estímulo y regocijo para quienes lo aceptan y practiquen voluntariamente" (1950: 5).

En las páginas del folleto puede leerse que este tipo de actividad consistía en una serie de ejercicios breves que se realizaban con la misma indumentaria de trabajo, siendo interpretado como: 
un alivio contra el sedentarismo, un estimulante para la prosecución de sus tareas, y un descanso psicomental de extraordinario poder para insistir en su insistencia y continuidad, la salud del empleado, la conservación de sus aptitudes laborativas y la eficacia de su rendimiento. (1950: 6).

Una Argentina en movimiento a través de la regulación de los cuerpos parece ser el principal propósito de Gimnasia de oficinas. En las páginas de la publicación se abogaba por la difusión de esta particular forma de actividad física. "Experimentarla aunque más no fuera por espíritu de ensayo, crítica o humorismo (tan característico de la idiosincrasia de nuestro pueblo) significaría a la postre su adopción sincera y entusiasta" (1950: 6). Se dejaba en claro que no buscaba imponerse esta nueva metodología, sino aplicarse por consenso, a tono con el adagio peronista que sostiene que gobernar es persuadir. Para ello se enumeraron las ventajas que proveía. Para el empleado creaba el hábito de una buena postura, contribuía a preservar el buen estado físico, eliminaba los errores y falta de atención para con el trabajo cotidiano. También colaboraba con las relaciones personales al interior de la oficina, ya que "hace menos frecuentes las discusiones o polémicas suscitadas por la intoxicación progresiva que produce el trabajo continuado y monótono, a la par que fomenta la cortesía, la camaradería y la comprensión entre empleados y jefes" (1950: 7).

Para el empleador las gratificaciones derivadas de la implementación de este método eran, como se mencionó, que se favorecían las relaciones con los subordinados, aumentando la calidad y cantidad de trabajo y mejorando significativamente la disciplina dentro del ambiente laboral. Por último, para el Consejo Nacional de Educación Física, la gimnasia de oficinas contribuía con el desarrollo del país en general ya que aumentaba la producción y disminuía el ausentismo. Estos temas fueron claves dentro de la relación entre el gobierno peronista y los empresarios, como lo ha demostrado Claudio Bellini (2014) ${ }^{3}$.

Asimismo, se hizo hincapié en la necesidad de formar líderes de oficinas que se seleccionarían entre los propios trabajadores. Estos aspirantes debían reunirse dentro del horario laboral por espacio de una hora con la finalidad de conocer el procedimiento.

Imagen 5:. Líderes de oficinistas, 1950.

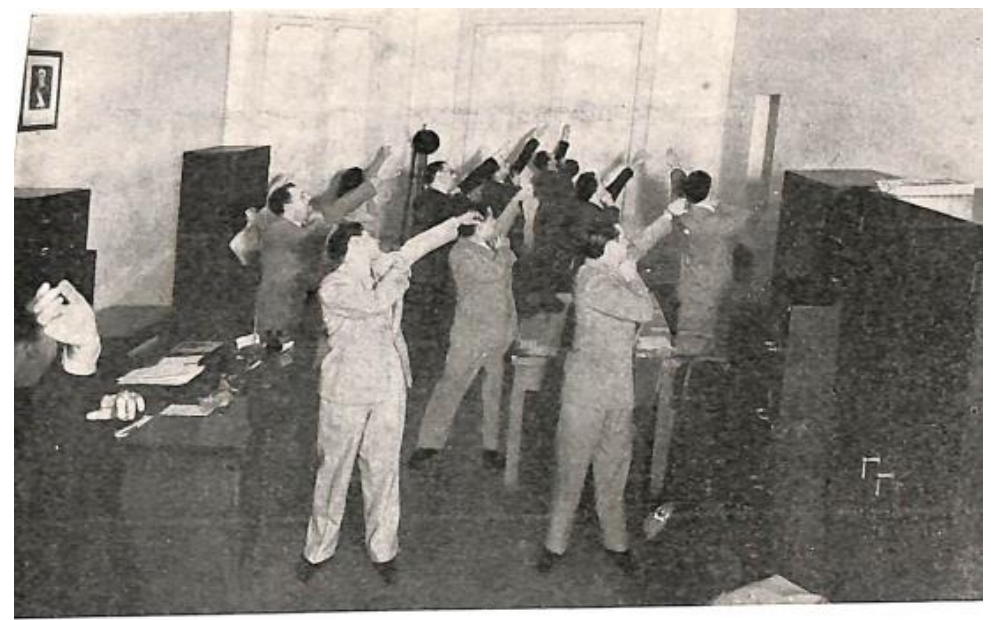

Conjunto de oficinites del Consejo Nacional de Falueación Fisica practieando un plan de este método

Fuente: Cuadernillo Gimnasia de Oficinas. 1950. 
Las clases debían durar entre cinco y diez minutos y debían hacerse efectivas en el momento en que:

\begin{abstract}
con mayor evidencia se evidencia el deseo de hacer una pausa en la tarea habitual: ya para reponer energías, procurar un descanso para la vista o la espalda dolorida, proporcionar libertad de acción a los miembros inferiores $u$ otras regiones musculares que han permanecido inmóviles y, sobre todo, distraer la mente absorbida por las preocupaciones de un trabajo por lo general monótono, y a esa altura de la jornada, deprimente y aburrido. (Consejo Nacional de Educación Física, 1950: 7).
\end{abstract}

Las ventajas que la gimnasia para oficinas promovía estaban ligadas a mejorar y fortificar la salud de los trabajadores. Asimismo, el progreso en la relación entre trabajadores y empresarios, así como el aumento en la producción se encontraban presentes en la base de la propuesta. Esto encuentra su conexión con palabras del primer mandatario: "Nosotros hemos sido los primeros en pensar que más vale cuidar bien a un hombre que a una máquina, porque entendemos que si una máquina vale mucho dinero, el hombre, económicamente considerado, también vale mucho dinero" (Perón, 1955: 7).

Los conceptos vertidos por el entonces presidente demuestran que la gimnasia para oficinas fue parte de un plan que contempló extender la educación corporal a vastos segmentos poblacionales. No obstante, al igual que con la gimnasia compensatoria en el aula, su implementación fue dificultosa. En un caso, por las resistencias docentes, en el otro por la dificultad del Estado en avanzar en empresas privadas. De hecho, en las conclusiones del citado cuadernillo se enfatizó en los "escépticos" que seguramente existirían con la propuesta. Una elegante forma de dejar en claro que la teoría no siempre se condice con la práctica.

\title{
Conclusiones
}

Ya en los primeros años del gobierno encabezado por Juan Domingo Perón la presentación de iniciativas legislativas destinadas a incidir en la cultura física poblacional fue visible. El contraste entre la postura militar y la médica fue un reflejo de las tensiones entre los diversos grupos que apoyaban al primer peronismo lo que demuestra que este no era un bloque monolítico. La creación por decreto del Consejo Nacional de Educación Física, bajo dependencia castrense, permitió zanjar esos disimiles puntos de vista y fue el puntapié inicial para comenzar a llevar adelante una serie de políticas que buscaron aplicarse en todo el país.

Gimnasia compensatoria en el aula y Gimnasia de oficinas, fueron dos estrategias ideadas y aplicadas por el Consejo Nacional de Educación Física. Ambos cuadernillos, explicados e ilustrados metódicamente, buscaron expandir la educación corporal de un modo del que poco se conocía hasta entonces en Argentina: en cualquier clase escolar y en las oficinas. Fue así que los aspectos pedagógicos de la Educación Física fueron considerados relevantes para formar el carácter de los ciudadanos de la Nueva Argentina. 


\section{Bibliografía}

Armus, D. (2016). Eugenesia en Buenos Aires: discursos, prácticas, historiografía. História, Ciências, Saúde, v.23, n³, 149-169.

Ball, S. (1990). Politics and policy making in education. Explorations in policy sociology. Londres, Inglaterra: Routledge.

Bellini, C. (2014). Convenciendo al capital. Peronismo, burocracia, empresarios y política industrial. Buenos Aires, Argentina: Imago Mundi.

Crisorio, R. (2007). Educación Física y biopolítica. Temas y matices, n¹1, 25-41.

Foucault, M. (1992). Microfísica del poder. Madrid, España: La Piqueta.

Galak, E. (2012). Del dicho al hecho (y viceversa). El largo trecho de la construcción del campo de la formación profesional de la Educación Física en Argentina. (Tesis de Doctorado en Ciencias Sociales). Universidad Nacional de La Plata, La Plata.

Galak, E, Orbuch, I. (2015). La educación de los cuerpos "peronistas". Un estudio a través de la prensa local de la zona metropolitana sur. Historia Educación Anuario, vol.16, no.2, p-90-105.

Galak, E. Orbuch, I. (2017). Cine, educación y cine educativo en el primer peronismo. El caso del Departamento de Radioenseñanza y Cinematografía Escolar. Revista Cinedocumental $\mathrm{n}^{\circ} 16$, pp. 120-141.

Parenti, C. (2015). Prólogo. Biografía de Alejandro J. Amavet y estudio esquemático de sus principales producciones. En: Ron, O.; Levoratti, A., coordinadores. Diálogos con Alejandro Amavet: A propósito de la educación física renovada. La Plata, Argentina: UNLP. Facultad de Humanidades y Ciencias de la Educación.

Puiggrós, A, y Bernetti, J. (1993). Peronismo: Cultura política y educación (1945-1955). Tomo V. Buenos Aires, Argentina: Galerna.

Scharagrodky, P (2006). En el nombre del Padre, del Hijo y del Espíritu Gimnástico: prácticas corporales, masculinidades y religiosidad en los Exploradores de Don Bosco en la Argentina de principios de siglo XX, en Revista Educar, Volumen 1, № 33, 56-72.

Somoza Rodríguez, M. (2006). Educación y Política en Argentina (1946-1955). Buenos Aires, Argentina: Miño y Dávila.

\section{Fuentes primarias:}

Cámara de Diputados. (1946a). Proyecto de creación de la Dirección Nacional de Educación Física. Recuperado de https://apym.hcdn.gob.ar/expedientes

Cámara de Diputados. (1946b). Proyecto de creación del Consejo Nacional de Educación Física y Medicina del Deporte. Recuperado de https://apym.hcdn.gob.ar/expedientes

Cámara de Diputados. (1947a). Consejo Nacional de Educación Física. Recuperado de https://apym.hcdn.gob.ar/expedientes 
Cámara de Diputados. (1947b). Inclusión entre los proyectos a tratarse el proyecto de ley sobre creación del Consejo Nacional de Educación Física. Recuperado de https://apym.hcdn.gob.ar/expedientes

Consejo Nacional de Educación Física. (1949). Gimnasia Compensatoria en el aula. Argentina.

Consejo Nacional de Educación Física. (1950). Gimnasia de oficinas. Argentina.

Perón, J. (1955). Discurso en el acto de clausura de la II Conferencia Nacional de Delegados Deportivos realizado por la Fundación Eva Perón. Buenos Aires: Subsecretaría de Informaciones.

\section{Notas}

\footnotetext{
${ }^{1}$ Véase Galak, E. Orbuch, I. (2017). Cine, educación y cine educativo en el primer peronismo. El caso del Departamento de Radioenseñanza y Cinematografía Escolar. Revista Cinedocumental, pp. 120-141.

2 De acuerdo a Carlos Parenti (2015), en el año 1928 egresó como profesor de Educación Física del Ejército Argentino, cursando sus estudios en la Escuela Militar de Gimnasia y Esgrima del Ejército, institución a cargo de la formación profesional de militares en la especialidad. Con posterioridad ocupó diversos lugares institucionales vinculados a la Educación Física durante el peronismo, y fue uno de los impulsores de la creación del Profesorado Superior Universitario de Educación Física en la Universidad Nacional de la Plata.

${ }^{3}$ En Convenciendo al capital. Peronismo, burocracia, empresarios y política industrial. Buenos Aires: Imago Mundi.
} 